Cahiers $d u$ MONDE RUSSE

\section{Cahiers du monde russe}

Russie - Empire russe - Union soviétique et États indépendants

$43 / 2-3 \mid 2002$

Contacts intellectuels, réseaux, relations internationales

\title{
Science et politique : les réseaux du Dr António Ribeiro Sanches (1699-1783).
}

\section{Georges DULAC}

\section{OpenEdition}

\section{Journals}

Édition électronique

URL : https://journals.openedition.org/monderusse/8492

DOI : 10.4000/monderusse.8492

ISSN : $1777-5388$

Éditeur

Éditions de l'EHESS

\section{Édition imprimée}

Date de publication : 1 avril 2002

Pagination : 251-274

ISBN : 2-7132-1781-4

ISSN : $1252-6576$

Référence électronique

Georges DULAC, «Science et politique : les réseaux du Dr António Ribeiro Sanches (1699-1783). », Cahiers du monde russe [En ligne], 43/2-3 | 2002, mis en ligne le 01 janvier 2007, consulté le 04 septembre 2022. URL : http://journals.openedition.org/monderusse/8492 ; DOI : https://doi.org/ 10.4000/monderusse.8492 
chercher : repérer : avancer

Cet article est disponible en ligne à l'adresse :

http://www.cairn.info/article.php?ID REVUE=CMR\&ID NUMPUBLIE=CMR 432\&ID ARTICLE=CMR 4320251

Science et politique : Ies réseaux du Dr António Ribeiro Sanches (1699-1783)

par Georges DULAC

Editions de l'EHESS | Cahiers du monde russe

2002/2-3 - Vol 43

ISSN 1252-6576 | ISBN 2713217814 | pages 251 à 274

Pour citer cet article :

_DULAC G. , Science et politique : les réseaux du Dr António Ribeiro Sanches (1699-1783), Cahiers du monde russe 2002/ 2-3, Vol 43, p. 251-274.

Distribution électronique Cairn pour les Editions de l'EHESS.

(C) Editions de l'EHESS. Tous droits réservés pour tous pays.

La reproduction ou représentation de cet article, notamment par photocopie, n'est autorisée que dans les limites des conditions générales d'utilisation du site ou, le cas échéant, des conditions générales de la licence souscrite par votre établissement. Toute autre reproduction ou représentation, en tout ou partie, sous quelque forme et de quelque manière que ce soit, est interdite sauf accord préalable et écrit de l'éditeur, en dehors des cas prévus par la législation en vigueur en France. Il est précisé que son stockage dans une base de données est également interdit. 


\section{SCIENCE ET POLITIQUE : LES RÉSEAUX DU DR ANTÓNIO RIBEIRO SANCHES (1699-1783)}

La constellation de relations dont ce médecin portugais s'est progressivement entouré tout au long de son existence est sans doute une des plus étonnantes de l'époque : du moins elle apparaîtrait telle si ses correspondances étaient toutes localisées ${ }^{1}$, ce qui est loin d'être le cas, et si ses archives personnelles ${ }^{2}$ livraient toutes les informations qu'elles recèlent. L'intérêt des écrits de Sanches, et notamment de ceux de langue française qui sont consacrés à la Russie et restent pour la plupart inédits, justifierait que de telles recherches soient menées, pour accompagner la préparation d'une édition qui viendra compléter celles qui ont été données de ses œuvres en portugais ${ }^{3}$. La présentation que je ferai ici de ce que j'ai appelé ses réseaux ne sera qu'une esquisse, dessinée sommairement à partir de quelques

1. Deux volumes de la Bibliothèque nationale de Vienne contiennent l'essentiel de la correspondance passive de Sanches aujourd'hui conservée, ou du moins localisée (ms 12713-12714). Des notes portées sur les manuscrits par le Dr Charles Andry, l'ami de Sanches qui a rassemblé ces lettres, confirment que la collection n'est en aucune façon complète, car certaines séries ont été rendues, parfois partiellement, à leurs auteurs. D'importantes séries de lettres de Sanches sont conservées à Pétersbourg (Archives de l'Académie des sciences et Bibliothèque nationale de Russie), à Berlin (Staatsbibliothek), à Evora (Bibliothèque publique), etc. ; ses journaux et cahiers de notes, notamment ceux conservés à Paris et à Madrid, contiennent en outre des minutes et des résumés de lettres ou de documents qu'il a envoyés à divers correspondants (voir la note suivante).

2. Ces archives sont importantes, mais dispersées entre Paris (Bibliothèque de la faculté de médecine, ms 41-43, 2015-2020), Madrid (Bibliothèque nationale, ms18370-18374), Braga (Bibliothèque publique, ms 640) et Moscou (Archives d'actes anciens, Rossijskij Gosudarstvennyj Arhiv Drevnih Aktov - RGADA, f. 1261-Voroncov, op. 1, n 2837, 2781, 2782, 2783, 2784). Des manuscrits de textes français de Sanches consacrés à la Russie ont été retrouvés par João Miranda aux archives des Affaires étrangères à Lisbonne (Arquivo nacional da Torre do Tombo, MNE).

3. António Nunes Ribeiro Sanches, Joaquim de Carvalho, ed., Obras, Université de Coimbra, t. 1,1959 ; t. 2, 1966 ; Ribeiro Sanches, Dificuldades que tem um reino velho para emendar-se e outros textos, Victor de Sá, ed., s. 1., Livros Horizonte, 1980. 
exemples, car l'espace me manquerait pour en proposer une analyse complète, qui dépasserait d'ailleurs ma compétence. Mais puisqu'ils n'ont jamais, à ma connaissance, été considérés dans toutes leurs dimensions, il m'a paru utile de donner un aperçu de la manière dont ils se sont constitués et des fonctions qu'ils ont remplies ${ }^{4}$.

S'agissant de Sanches, il m'a paru préférable de parler de réseaux, au pluriel, parce que cette image un peu fourre-tout me semble ainsi correspondre plus exactement à ce que je voudrais évoquer : chacun d'eux peut être défini comme un ensemble de relations durables et actives, qui a sa propre cohérence et se trouve plus ou moins séparé des autres. Ainsi on pourra considérer qu'en Russie Sanches entretient deux réseaux, l'un dans le milieu académique, l'autre à la cour et dans la grande noblesse : entre ces deux mondes, il existe peu de ressemblances et d'intérêts communs, malgré les quelques passerelles qui les relient.

Trois pays tiennent une place essentielle dans les réseaux de Sanches: le Portugal, qu'il a quitté définitivement en 1726, à l'âge de vingt-sept ans, mais avec lequel il a toujours conservé des rapports très actifs ; la Russie, où il a mené pendant dix-sept ans, de 1731 à 1747, une brillante carrière, qu'il a conclue avec la charge de médecin de l'impératrice Élisabeth : un pays qui, à l'égal du Portugal, a été au centre de sa réflexion politique et avec lequel il a gardé de nombreuses relations jusqu'à la fin de sa vie ; enfin la France, où il a vécu après son départ de Russie, faisant fructifier une expérience exceptionnellement étendue des sociétés et des foyers intellectuels européens, que de multiples échanges lui ont permis d'enrichir et de tenir à jour jusqu'à ses dernières années. Il faut ajouter que les Russes et les Portugais séjournant hors de leur pays, et parfois définitivement exilés, ont toujours tenu une place importante dans ses amitiés et ses travaux, peut-être parce que plusieurs d'entre eux étaient comme lui des intermédiaires particulièrement zélés, mais trop attachés à leur ouverture sur le monde pour accepter un repli sur leur pays d'origine : à la manière du prince Dmitrij Alekseevič Golicyn ${ }^{5}$, qui, arrivé en France à vingt-deux ans, se proclamait quelques années plus tard son disciple et ne devait jamais retourner en Russie, sinon pour quelques semaines en 1769 , peu après son mariage. Cependant un des objets de mon étude sera de montrer qu'on ne peut limiter au Portugal, à la Russie et à la France la géographie des relations de Sanches, qui, tout en s'étendant à une dizaine de pays, ont formé autour de notre médecin un véritable ensemble, possédant une sorte d'unité organique.

4. Sur Sanches, voir notamment Maximiano Lemos, Ribeiro Sanches, a sua vida e a sua obra, Porto, Tavares Martins, 1911 ; David Willemse, António Nunes Ribeiro Sanches - élève de Boerhaave - et son importance pour la Russie, Leyde, Brill, 1966. Je voudrais exprimer ma gratitude pour l'aide que m'a apportée João Miranda, qui m'a communiqué de nombreux documents et publications : la bibliographie portugaise consacrée à Sanches est très abondante et je n'en mentionnerai ici qu'une petite partie. Je remercie également Mme Marina Reverseau, qui m'a aidé à tirer parti de la bibliographie russe, et Sergej Karp, qui a bien voulu relire cet article.

5. Voir Georges Dulac et Ludmilla Evdokimova, « Politique et littérature. La correspondance de Dmitri A. Golitsyn, 1760-1784 », Dix-huitième siècle, 22, 1990, p. 367-400 ; Dmitri Alekseevitch Golitsyn, «Lettres sur le servage et le développement de la Russie, 1765-1771 », ed. Georges Dulac, in., Politique et économie au temps des Lumières, études réunies par Gérard Klotz, Saint-Etienne, Publications de l'Université de Saint-Etienne, 1995, p. 117-161 (Lire le Dix-huitième siècle). 
Notons enfin qu'il aurait été possible d'étudier cet ensemble de manière à peu près complète à partir de la seule période française de la vie de Sanches (17471783), parce que c'est durant ces années que les réseaux qu'il a constitués ont atteint à la fois leur plus grande extension et leur plus forte activité. Mais le choix de cette perspective aurait conduit à en donner une vision à peu près statique, alors que leur exceptionnelle ampleur tient à un processus d'accumulation progressive qui a débuté trente ans plus tôt. Je commencerai donc par proposer une vue cavalière de quelques-unes de ses relations les plus caractéristiques en les saisissant à leur origine, au cours des quatre principales périodes de sa vie. J'en viendrai ensuite à examiner de plus près les causes de leur développement, c'est-à-dire les principales fonctions qu'elles ont pu assumer.

\section{La constitution des réseaux de Sanches (1716-1747)}

\section{Au départ, le Portugal}

De la première époque de la vie de Sanches, celle qui précède son départ du Portugal en 1726, datent plusieurs relations qui caractérisent son attachement à un pays qu'il appellera toujours sa «patrie». Ce sont en premier lieu quelques connaissances avec qui il maintient des liens solides, comme par exemple un de ses amis de Benavente (la petite ville proche de Lisbonne où il avait exercé la médecine), Manuel Pacheco Sampaio Valladares (1673-1737), à qui il adresse une série de lettres en 1733-1735, depuis Moscou et Pétersbourg ${ }^{6}$ : à ce vieil homme de lettres, qui semble avoir été un esprit ouvert et tolérant, il confie de nombreux détails sur les principaux épisodes de son existence depuis son exil, l'évolution de ses opinions religieuses et plusieurs aspects de son activité en Russie. Valladares, qui s'était livré notamment à des travaux historiques, lui facilitera l'acquisition de livres portugais, qui concernent par exemple l'université de Coimbra, l'histoire du Portugal ou encore la Chine : comme nous le verrons, le choix de ces livres était en rapport avec certains intérêts persistants de Sanches. Il est question dans ces lettres à Valladares de plusieurs autres de ses connaissances au Portugal, mais on notera surtout qu'il y évoque en 1735 les initiatives qu'il prend pour établir des relations entre l'Académie des sciences de Pétersbourg et l'Academia Real de Historia de Lisbonne (fondée en 1720, elle laissera la place en 1779 à l'Académie des sciences du Portugal). À sa demande, le président de l'Académie impériale, le baron Johann Albrecht von Korff, et son secrétaire, Christian Goldbach, un ami de Sanches, se prêteront à l'envoi d'un présent à l'Académie portugaise, une douzaine de volumes magnifiquement reliés des travaux de la compagnie pétersbourgeoise, qui en 1738

6. Maximiano Lemos, « Cartas de Ribeiro Sanches ao Dr Pacheco Valladares », Archivos de historia da medicina portugueza, Nova série, vol. 2, 1911, p. 111-120, 150-156, 193-196 ; vol. 3 , 1912, p. 28-30, 40-48, ...-80, 131-139 ; vol. 4, 1913, p. 25-31, 57-62, 90-96, 119-128, 137-143 (nous n'avons pu consulter qu'un exemplaire incomplet). 
recevra de son côté une collection d'ouvrages portugais ${ }^{7}$. Ces échanges devaient se poursuivre les années suivantes. Sanches fut probablement en relations à ce propos avec deux membres éminents de l'Académie d'histoire qu'il nomme à Valladares, D. Martinho de Mendonça de Pina, un familier du palais royal, auteur d'un ouvrage sur l'éducation des jeunes nobles (Sanches aurait écrit pour lui un mémoire sur l'hygiène des enfants), et le père $\mathrm{D}$. Luis Caetano de Lima, un théatin qui avait été chargé de missions politiques et diplomatiques. Ces diverses circonstances, qui prouvent que Sanches n'a jamais perdu contact avec son pays natal, contribuent également à expliquer qu'il y ait été quelques années plus tard une personnalité connue, d'autant plus qu'à ses relations anciennes devaient s'en ajouter d'autres, à la suite de rencontres intervenues à l'étranger, et notamment à Paris. C'est ainsi que Diogo Barbosa Machado, de l'Académie d'histoire de Lisbonne, lui demandera en 1757 une notice sur sa vie et ses œuvres ${ }^{8}$ qu'il utilisera dans un copieux article paru en 1759 dans un supplément de sa Bibliotheca Lusitana (t. 4, p. 56-58).

Signalons dès maintenant qu'une autre amitié de jeunesse devait contribuer au développement d'un ensemble de relations que Sanches cultivera depuis Pétersbourg avec les missionnaires jésuites de Pékin. En 1716 et les années suivantes, il avait en effet fait ses humanités au Collège des arts de Coimbra, tenu par les jésuites, en compagnie de Polycarpo de Sousa, qui devait partir pour la Chine en 1725, après d'autres missionnaires ayant reçu une formation analogue, comme André Pereira, qui allait également être du nombre de ses correspondants. Le premier devait être investi en 1743 comme troisième évêque de Pékin, tandis qu'André Pereira, qui allait être emporté par une épidémie à la fin de cette même année, était provincial de jésuites de Chine ${ }^{9}$ : nous les retrouverons à propos des relations scientifiques dont Sanches s'entourera en Russie.

\section{Une période d'errance}

La seconde époque à considérer dans la constitution des réseaux de Sanches correspond dans son existence à une période d'incertitude, voire d'errance, qui se situe entre son départ du Portugal et son installation en Russie, cinq ans plus tard : des années dont la chronologie et l'emploi exacts nous échappent en partie, mais qui devaient

7. João Miranda, «A. N. Ribeiro Sanšes i russko-portugal’skie svjazi v XVIII v.» (A. N. Ribeiro Sanches et les relations russo-portugaises au XVIII' siècle), Vestnik Moskovskogo universiteta, Serija 8, Istorija, 3, 1987, p. 41-51 et notamment p. 50-51 ; voir également Protokoly zasedanij konferencii imp. Akademii nauk s 1725 do 1803 (Procèsverbaux des séances de l'Académie impériale des sciences de 1725 à 1803), Saint-Pétersbourg, 1897-1911, 4 vol., t. 1, p. 203-204 (2 juin 1735). Nous renverrons à cette édition des procèsverbaux des séances de l'Académie en abrégeant la référence en Procès-verbaux.

8. Madrid, ms 18372, fol. 243-244.

9. Arthur Viegas, «Ribeiro Sanches e o P. Polycarpo de Sousa, terceiro bispo de Pekim », Revista de historia, vol. 10, 1921, p. 241-263 ; João Miranda, « A missionação portuguesa e a Rússia nos séculos XVII e XVIII », in Congresso internacional de historia. Missionação portuguesa e encontro de culturas, Actas, vol. 3, Braga, 1993, p. 103-122. 
s'avérer extrêmement fécondes parce qu'elles lui permirent, au cours de séjours dans plusieurs centres intellectuels d'Europe occidentale, d'accumuler expériences et observations tout en amorçant des relations qui allaient avoir de nombreux prolongements. Sanches passe alors un an à Londres, où il se convertit au judaïsme, apprend l'anglais et les mathématiques, et se familiarise avec la médecine anglaise qu'il jugera supérieure à bien des égards à celle du continent (on sait que l'inoculation était largement répandue en Grande-Bretagne dès 1730) ; il voyage en France, entre en contact avec des médecins de Montpellier et de Marseille, puis séjourne quelques mois à Livourne, à Pise et à Turin, dont il lui arrivera de vanter l'université, avant de gagner Leyde, où il suivra les cours de Herman Boerhaave pendant plus d'un an.

À Londres, il se lie notamment avec des exilés portugais, qui sont pour la plupart des marranes, comme le médecin Jacob de Castro Sarmento et le minéralogiste Mendes da Costa. Castro Sarmento (1691-vers 1760), qui avait étudié à Coimbra vers 1717 et s'était installé à Londres dès 1721, y avait publié l'année suivante une brochure en faveur de l'inoculation. Il avait poursuivi des travaux de médecine, de physique et de chimie et devait devenir membre de la Royal Society en 1730, puis professeur à l'université d'Aberdeen en 1736. Il semble qu'il soit resté jusque dans les années 1750 un des principaux correspondants de Sanches en Grande-Bretagne, un de ceux qui l'informaient des nouvelles publications et contribuaient à le fournir en livres et en brochures, dont il enregistrait parfois l'arrivée dans ses cahiers par ballots entiers : qu'il s'agisse de médecine, d'économie politique, de philosophie ou d'histoire, la production intellectuelle britannique devait en effet rester au premier plan de ses intérêts, comme en témoignent à la fois ses notes personnelles, ses écrits sur la Russie, qui sont truffés de références à Hume et aux penseurs de l'école historique écossaise, et le catalogue de sa bibliothèque ${ }^{10}$. Castro Sarmento de son côté dut sans doute à Sanches certains contacts avec l'Académie de Pétersbourg, et nous verrons qu'il apporta également sa contribution aux échanges que celui-ci entretenait avec les savants de Pékin. Cependant il est probable que le séjour londonien de Sanches lui acquit d'autres relations durables : mais nous ignorons s'il faut par exemple faire remonter à cette époque celles qu'il avait vers 1744 et au cours des années suivantes avec le botaniste et physicien Peter Collinson (1694-1768), qui fut associé à sa correspondance avec J. G. Gmelin et avec les missionnaires jésuites ${ }^{11}$. Quoi qu'il en soit, un autre savant portugais, d'origine noble, João Jacintho de Magalhães (1722-1790), qui s'exila vers 1756 et, après avoir séjourné en France et en Hollande, s'installa définitivement en Angleterre en 1764, allait à son tour et pendant de longues années procurer régulièrement à Sanches des publications anglaises ${ }^{12}$. Nous aurons à reparler de cet ami très proche

10. Catalogue des livres de feu M. Sanchès, Paris, Debure, 1783 (reproduit à la fin de D. Willemse, op. cit.).

11. Voir les lettres de Sanches à Johann Georg Gmelin conservées à Berlin, Staatsbibliothek, collection Darmstaedter (Darmst 3 i 1750 (1)), notamment celle datée de Moscou, 3 avril 1744 (fol. $\left.10-11 \mathrm{v}^{\circ}\right)$.

12. Ricardo Jorge, « Amigos de Ribeiro Sanches, J. H. de Magellan », A Medicina contemporânea, 1, 1910, p. 3-6, 11-14. 
de notre médecin, qu'on appelait Magellan hors du Portugal : physicien de réputation internationale et spécialiste de l'instrumentation scientifique, il devait luimême constituer un réseau très étendu de correspondances scientifiques qui s'étendait dans les années 1770 du Portugal à la Russie.

Nous sommes mal renseignés sur le séjour que Sanches fit en Italie vers 1728 ou 1729, ainsi que sur les relations qui trouvèrent peut-être leur origine durant cette période, et que des recherches d'archives pourraient éventuellement éclairer. Sans avoir l'importance de la Grande-Bretagne dans sa vie intellectuelle, ce pays devait pourtant y tenir une place remarquable, comme l'atteste le contenu de sa bibliothèque, surtout en ce qui concerne la politique et l'économie : Franco Venturi a noté qu'on y trouvait toutes les œuvres les plus caractéristiques des lumières italiennes, du Vero despotismo de Gorani à l' Economia politica de Pietro Verri ${ }^{13}$. D'autre part ses écrits politiques et ses archives contiennent des références à certaines institutions qu'il a pu observer, comme le port franc de Livourne, qu'il propose en modèle aux Portugais, en vantant la politique de tolérance des derniers Médicis et ses heureux effets sur l'économie et la population de la région; ou encore, de façon plus surprenante, l'université de Turin, qu'il recommande en 1756, en concurrence avec Strasbourg, au vice-chancelier Mihail Voroncov pour l'éducation d'un de ses parents $^{14}$ : il précise à cette occasion qu'il sera en mesure de recommander le jeune homme à des amis qui veilleraient sur ses études; mais nous ignorons tout des correspondants qu'il pouvait avoir dans cette ville. Dans une de ses lettres à Valladares, il évoque d'autre part les quelques mois qu'il avait passés à l'université de Pise et surtout les fructueuses discussions qu'il avait eues alors avec Alberto De Soria. Ce professeur de médecine et de philosophie, qui devait achever d'éloigner Sanches du judaïsme, était déiste et newtonien, et comme beaucoup de ses collègues de l'université, il avait de nombreux contacts avec l'Angleterre et la Hollande. Rien n'indique cependant que Sanches ait conservé après son départ des rapports avec ce milieu. Les correspondances provenant d'Italie qui subsistent parmi ses papiers proviennent de Portugais et sont assez peu fournies, à l'exception de celle qu'il a reçue de son frère Marcello : installé comme chirurgien à Naples en 1765, celui-ci semble y avoir végété au milieu de grandes difficultés, bien que de Paris son aîné lui ait conseillé de se faire appuyer par l'abbé Galiani, à qui il avait dû le recommander. Bien que nous ne puissions en juger que par deux lettres ${ }^{15}$ et que nous en ignorions l'origine et la durée, il faut surtout remarquer la relation très amicale que Sanches paraît avoir entretenue dans les années 1750 avec Luís António Verney (1713-1794), auteur d'un célèbre ouvrage attaquant l'enseignement des jésuites, Verdadeiro método de estudar para ser util a República, publié à Naples en 1746. Né à Lisbonne, Verney s'était fixé à Rome, et c'est de cette ville qu'il écrit à Sanches des lettres où il se plaint des persécutions incessantes qu'il

13. Franco Venturi, Settecento riformatore, IV, t. 1, I grandi stati dell'Occidente, Turin, Einaudi, 1984,p. 227.

14. Madrid, ms 18373 , fol. $15-21 \mathrm{v}^{\circ}$.

15. Vienne, ms 12713. Verney écrit à Sanches en latin. 
subit de la part des jésuites, qui ont publié contre lui un pamphlet où il est, écrit-il, traité aussi férocement que l'était Spinoza ${ }^{16}$. Son correspondant aura lui-même pour préoccupation constante de dénoncer la malfaisance politique des systèmes éducatifs placés sous l'influence du clergé catholique, et tous deux devaient être parmi les principaux inspirateurs des réformes de Pombal dans ce domaine.

Dernière étape des voyages de Sanches avant son départ pour la Russie, le séjour qu'il effectue en Hollande d'avril 1730 à juillet $1731^{17}$ est à l'origine de deux des réseaux les plus importants qu'il développera par la suite : l'un a pour noyau les disciples de Boerhaave dont il est venu suivre l'enseignement à Leyde et auquel il vouera toujours un véritable culte; l'autre aura pour point de départ les relations qu'il noue à La Haye avec l'ambassadeur du Portugal, D. Luis da Cunha (16621749), membre de l'Académie d'histoire de Lisbonne et penseur politique éclairé, considéré comme le maître du futur marquis de Pombal. Sanches, qui devait le retrouver à Paris en 1748, put lire son Testamento politico ${ }^{18}$ qui connut une diffusion manuscrite avant d'être publié au début du XIXe siècle. Da Cunha était de ces politiques portugais qui voulaient relever la puissance de leur pays en modernisant l'État et le système éducatif, tout en favorisant un renouveau économique, ce qui impliquait la réduction de l'influence de l'Église. C'est en Hollande qu'à sa demande Sanches écrivit un projet de réforme des études médicales au Portugal, qu'il devait reprendre à Paris pour le gouvernement de Pombal (Metodo para aprender e estudar a medecina, 1763). À cette époque, il était une personnalité bien connue des milieux diplomatiques portugais, avec lesquels il ne devait pas cesser d'avoir des relations jusqu'à la fin de sa vie.

Les correspondances que Sanches a entretenues avec deux des disciples les plus connus de Boerhaave, Hieronym David Gaubius ${ }^{19}$ et Gerard Van Swieten, sont parmi les plus fournies qui nous soient parvenues ${ }^{20}$. Toutes deux, qui commencèrent pendant son séjour en Russie, se prolongeront après son installation à Paris, et même, dans le cas de Gaubius, jusqu'aux dernières années du médecin de Leyde, qui en 1777 écrira qu'il voyait en Sanches «un des [ses] meilleurs amis ». Un des aspects les plus intéressants de cette dernière correspondance réside dans les diverses relations qu'elle révèle, au delà du dialogue ordinaire sur des matières médicales : par exemple quand Sanches, en 1768, envoie un jeune Portugais, Felix Antonio Castrioto, suivre l'enseignement de Gaubius, puis lui demande de faciliter son engagement au service de la Russie (ce qui d'ailleurs ne se fera pas, le candidat ayant finalement révélé d'autres intentions); ou lorsque

16. En juillet 1771, Sanches note dans son journal que Verney a été banni des États du Pape et accompagne cette nouvelle d'un éloge de ses ouvrages (Paris, ms 2015, fol. 108 rº).

17. David Willemse, op. cit., notamment p. 64 sq.

18. Il en note des extraits dans un de ses cahiers en septembre 1756 (Madrid, ms 18372, fol. 228).

19. Sophia Wilhelmina Hamers-van Duynen, Hieronymus David Gaubius (1705-1780) zijn correspondentie met Antonio Nunes Ribeiro Sanches, Amsterdam, Van Gorcum, 1978.

20. Vienne, ms 12713. 
Gaubius évoque en 1776 une séance d'expérimentation à laquelle il a participé avec deux autres amis de Sanches, le prince D. A. Golicyn et Magellan, dans le laboratoire du prince, à La Haye. La correspondance reçue de Van Swieten, qui commence en latin puis se poursuit en français à partir de 1741, est plus variée mais non moins amicale : on y trouve évoqués par exemple le recrutement souvent difficile de médecins pour la Russie, dont Sanches s'occupe depuis Pétersbourg en même temps que du transfert des manuscrits de Boerhaave, les circonstances de l'engagement de Van Swieten comme médecin de l'impératrice Marie-Thérèse et son arrivée à Vienne en 1745 ; ou des sujets plus généraux, tels que les idées de Sanches sur l'importance de l'histoire et des humanités dans les formations universitaires, la place croissante du français dans les échanges scientifiques, qui irrite Van Swieten, ou encore certaines expériences et théories de Réaumur, de Needham, de Buffon ou de Franklin ${ }^{21}$.

\section{La Russie}

Les très nombreuses relations que Sanches a nouées pendant les dix-sept années qu'il a passées en Russie, se répartissent, comme celles qu'il a amorcées en Hollande, en deux sphères bien distinctes : d'un côté des hommes de science, et surtout des membres de l'Académie de Pétersbourg; de l'autre, des personnages de la cour et de l'entourage impérial. Les unes et les autres se prolongeront souvent après son installation à Paris ${ }^{22}$. Ses archives personnelles prouvent que peu après son arrivée en Russie, il a commencé a fréquenter l'Académie impériale des sciences dont il devait être élu membre étranger au moment de quitter le pays : ce fut sans doute autant par nécessité professionnelle que par goût, car il avait été chargé de composer une pharmacopée et devait d'autre part passer par la librairie de l'Académie pour se procurer des livres. Il semble aussi que ses relations internationales déjà étendues, ainsi que sa bonne connaissance de l'anglais, aient été appréciées par la compagnie, car son nom apparaît souvent dans ses procès-verbaux de séances. Enfin on doit noter qu'au début des années 1740, alors qu'il occupe une très haute position à la cour, avec le grade de conseiller d'État actuel, il est en

21. Nous ne nous attarderons pas davantage sur les correspondances médicales de Sanches. En 1760, dans une lettre au Dr Joachim Pedro de Abreu, médecin de la cour de Lisbonne (Paris, ms 2019, fol. $168 \mathrm{v}^{\circ}$ ), il mentionnera, à propos de l'expérience qui lui permet de traiter de l'enseignement de la médecine, les correspondances qu'il a entretenues avec G. Van Swieten, H. D. Gaubius, A. von Haller, Johann Friedrich Schreiber (Pétersbourg) et Justus Gottfried Günz (Leipzig). Cette dernière, qui a dû commencer dans les années 1740 et a duré pendant plusieurs décennies, est bien représentée dans les archives de Sanches (Paris, ms 2019, Vienne, ms 12713 , etc.).

22. David Willemse, op. cit. ; Romulo de Carvalho, «Ribeiro Sanches na Rússia », Relações entre Portugal e a Rússia no século XVIII, Lisbonne, Sá da Costa, 1979, p. 17-53 ; João Miranda, «A. N. Ribeiro Sančes i russko-portugal'skie svjazi... », art. cit.; G. Dulac, «Ribeiro Sanches et le développement de la Russie : le travail politique d'un médecin encyclopédiste (1764-1771) », in Ici et ailleurs: le dix-huitième siècle au présent. Mélanges offerts à J. Proust, Tokyo, 1996, p. 393-421. 
mesure de se comporter en protecteur de certains académiciens (en soutenant par exemple, en 1743, les demandes d'augmentation de salaire de Gerhard Friedrich Müller et de Johann Georg Gmelin), et de l'Académie elle-même, alors privée pratiquement de direction et dans une situation qu'il juge déplorable. Parmi ses plus anciennes relations, on compte Leonhard Euler, dont il a fait la connaissance en 1732 et avec qui il correspond en 1740 à propos de l'application des mathématiques aux sciences sociales (les questions qu'il lui pose alors sont inspirées par les travaux d'arithmétique politique publiés en Grand-Bretagne). Il conservera avec lui des rapports amicaux, lui rendra visite à Berlin en 1747 et continuera à correspondre avec lui, puis avec son fils aîné Johann Albrecht après la seconde installation de la famille à Pétersbourg, en 1766. Ses relations avec J. G. Gmelin ${ }^{23}$ ont également été excellentes et leur correspondance se poursuivra après son départ : à partir de 1743, après la grande expédition en Sibérie, tout en abordant avec le naturaliste certaines questions scientifiques, Sanches échange avec lui divers services et quelques confidences, Gmelin par exemple s'occupant en son absence de ses commandes de livres tandis que le médecin de l'impératrice lui propose son aide pour sa correspondance avec les jésuites de Pékin : c'est souvent par le biais de telles relations personnelles que Sanches intervient dans les affaires d'une compagnie dont il est visiblement très proche, sans en faire partie ${ }^{24}$. Lorsque Gmelin, installé à Tübingen et peu désireux de retourner en Russie, sera en conflit avec l'Académie à propos de la publication de ses travaux, ils échangeront leurs commentaires sur la politique jalouse qu'elle pratique et dont est également victime leur ami commun Joseph Nicolas Delisle, que Sanches fréquente à Paris. Signalons enfin ses relations avec Jacob von Stählin, professeur d'éloquence et un des plus anciens membres de l'Académie, avec qui il entretiendra une correspondance amicale jusqu'à la fin de sa vie.

Comme nous l'avons déjà indiqué, c'est depuis Pétersbourg que Sanches développe des relations très actives avec les savants jésuites de Chine, son ancien condisciple Polycarpo de Sousa (1796-1757) et ses autres compatriotes, André Péreira (1689-1743), mandarin et vice-président du tribunal de mathématiques, et Domingos Pinheiro (1688-1748), mathématicien et astronome ; après la mort de

23. Aux lettres de Gmelin à Sanches conservées à Vienne (ms 12713), il faut ajouter les documents conservés à la Staatsbibliothek de Berlin, dans le Nachlass Gmelin et surtout dans la collection Darmstaedter (Darmst 3 i 1750 (1), fol. 1-61) : ce dernier dossier comprend vingt-neuf lettres et billets de Sanches à Gmelin des années 1743-1752. Sanches y fait état de relations régulières qu'il entretient dès 1744 avec Albrecht von Haller, alors à Göttingen, avec Peter Collinson, à Londres, avec les jésuites de Pékin, et plus tard avec Schöpflin, par l'intermédiaire de qui passent certaines lettres envoyées de Tübingen. Notons que dans sa lettre du 15 octobre 1749 (Vienne, ms 12713, fol. $349 \mathrm{r}^{\circ}-\mathrm{v}^{\circ}$ ), Gmelin parle de la « mésintelligence » survenue entre Sanches et Haller : « Ne croyez jamais, lui écrit-il notamment, qu'a Mr. Haller sont jamais venû de tels puerilités à la tete, de vous mepriser comme Juif. Le Sçavoir n'est pas attaché au Christianisme, comme l'ignorance n'est pas l'essence [lecture douteuse] du Judaisme, \& cet homme meriteroit bien du chatiment, qui mepriseroit le sçavoir d'un juif par raison qu'il seroit Juif. $[\ldots] »$.

24. Le 20 avril 1744, par exemple, Gmelin fait état au cours d'une séance de l'Académie d'une lettre qu'il a reçue de Sanches à titre privé (Procès-verbaux, t. 2, p. 16). 
Pereira, August von Hallerstein (1703-1774), astronome et cartographe, prendra l'initiative de participer à cette correspondance et, bien qu'originaire de Bohême, lui écrira en portugais ${ }^{25}$. Quand Sanches commença à fréquenter le milieu scientifique de Saint-Pétersbourg, l'Académie impériale amorçait tout juste des relations avec ces jésuites de Pékinn ${ }^{26}$, et le médecin portugais se lia rapidement d'amitié avec les deux hommes qui en avaient l'initiative, Gottlieb Siegfried Bayer, professeur de langues et histoires orientales, et surtout sinologue, et l'astronome et géographe Joseph Nicolas Delisle. Dès 1731, Bayer avait par exemple sollicité l'avis des révérends pères sur son Museum sinicum, publié l'année précédente à Pétersbourg. Malgré la difficulté d'acheminer les envois, qui étaient confiés aux caravanes commerciales russes, un courant d'échanges important s'établit, que Sanches contribua à faciliter et à renforcer par les correspondances personnelles qu'il entretenait avec ses compatriotes, parallèlement à celles de l'Académie. Ces dernières concernaient l'échange d'observations et de questions, ainsi que l'expédition de livres et de documents, tels que des cartes et des calendriers astronomiques, ou l'envoi de spécimens botaniques et de curiosités naturelles. En cette matière, la lourdeur de l'administration académique n'était pas le moindre des obstacles, et il arriva par exemple que le naturaliste Johann Georg Gmelin, désespéré de n'avoir pu faire prendre en compte ses demandes, s'adressât à son ami Sanches pour obtenir par son entremise des semences que pourraient lui fournir les savants de Pékin pour le jardin botanique ${ }^{27}$. Il faut observer que, tout comme les échanges académiques, ceux qu'entretenait Sanches prenaient fréquemment appui sur des relations internationales étendues : c'est ainsi qu'il put offrir aux jésuites astronomes deux instruments scientifiques acquis pour eux en Angleterre grâce à l'entremise de son ami Jacob de Castro Sarmento, membre de la Royal Society. Les longues lettres que Sanches recevait de Chine montrent que tout en collaborant à ces échanges proprement scientifiques, il pouvait aborder des sujets plus larges et plus variés, tels que la situation du christianisme dans ce pays, les difficultés rencontrées par Polycarpo de Sousa dans ses rapports avec Rome, ou les relations commerciales entre la Russie et la Chine : assez malencontreusement, semble-t-il, il tenta même, dans les années 1740, de faciliter les opérations du directeur de la caravane russe, Lorentz Lang, en lui procurant l'appui financier des révérends pères ${ }^{28}$. De leur côté les jésuites semblent avoir envisagé de tirer parti de la haute position que le médecin occupait à la cour de Pétersbourg pour faciliter leur éventuelle implantation en Russie. La dernière lettre de Polycarpo de Sousa à Sanches que nous possédons date de 1750, et nous ignorons si leurs relations se sont poursuivies. Toujours est-il que bien des années plus tard, alors que cette génération de missionnaires aura depuis longtemps

25. Paris, ms 2018, fol. 146-150 (1746).

26. E. S. Kuljabko, I. A. Perel'muter, « Iz naučnoj perepiski peterburgskih učenyh XVIII v. » (Extraits de la correspondance scientifique de savants de Pétersbourg du XVIII ${ }^{\mathrm{e}}$ siècle), in Strany i narody vostoka (Pays et peuples d'Orient), Moscou, Nauka, 1969, p. 136-152.

27. Gmelin à Sanches, 19 avril 1744 (Vienne, ms 12713, fol. 329).

28. J. Miranda, « A missionação... », art. cit. (1993), p. 120. 
disparu, Sanches servira encore d'intermédiaire, au moins occasionnellement, pour les échanges entre Pétersbourg et Pékin : entre novembre 1776 et mars 1777, il rend compte à son vieil ami Jacob von Stählin de la manière dont il s'acquitte de l'expédition, via Lorient et Canton ${ }^{29}$, d'une caisse de livres et d'estampes destinée au père Cibot.

Du côté de la cour et de l'entourage impérial, Sanches a également établi de nombreuses relations, successivement comme médecin des armées du maréchal Münnich (1735-1737), puis du Corps des cadets, et enfin comme médecin de l'impératrice (1740). Cependant les documents qui peuvent éclairer cet aspect de son activité sont rares et fragmentaires. Quelques traits généraux s'en dégagent cependant, qui permettent d'expliquer que plusieurs des personnages qu'il a fréquentés, le plus souvent placés au sommet de la société et de l'État, conserveront une réelle importance dans sa vie après son départ de Russie. Il convient de noter tout d'abord le très grand prestige dont il a joui comme médecin de la cour : déjà bien établie à l'avènement de l'impératrice Elisabeth en 1740, sa réputation sera encore rehaussée en 1744 lorsque la grande-duchesse Catherine lui devra son salut. Aussi ne faut-il pas s'étonner que la plupart des grands seigneurs russes séjournant à Paris aient recouru à ses services, comme en témoignent abondamment ses agendas, et qu'on lui ait fréquemment demandé des consultations à distance. En 1757 , le vice-chancelier Mihail Voroncov lui fera même avec insistance la proposition de revenir en Russie ${ }^{30}$. Cependant ses relations avec le vice-chancelier, ou celles, dont nous reparlerons, avec le comte Kirill Razumovskij, montrent que dans la haute société russe on ne l'appréciait pas seulement comme médecin, mais aussi comme un homme de pensée, tout particulièrement en matière d'éducation : tout deux lui demanderont des plans d'études, le premier vers 1756 pour un jeune parent de quinze ans, peut-être Aleksandr Romanovič Voroncov, le second dix ans plus tard pour ses fils, confiés à Jean-Daniel Schöpflin, à Strasbourg. À la même époque, Ivan Beckoj, qu'il avait dû connaître vers 1744 dans l'entourage de la grandeduchesse et de sa mère, lui demandera de collaborer à la définition des statuts des nouveaux établissements d'éducation, ce qui l'amènera à développer des réflexions sur l'ensemble des problèmes posés, selon lui, à la société russe ${ }^{31}$. On pourrait donner plusieurs autres exemples de la considération durable que Sanches s'était acquise dans de tels domaines, grâce notamment à une culture philosophique et politique qu'il avait commencé à développer dès avant son arrivée en Russie et qu'il ne cessera d'enrichir. Mentionnons seulement parmi les correspondances qui en témoignent, celles, très amicales, qu'il a entretenues avec Thimotheus von Klingstedt ${ }^{32}$, membre de la commission du commerce et auteur d'un ouvrage sur les

29. Pétersbourg, Bibliothèque nationale de Russie, fonds 871-Stählin, $n^{\circ} 664$, fol. 63-68.

30. La minute de la lettre de Sanches refusant les propositions très avantageuses du comte M. I. Voroncov est datée de février 1758 (Madrid, ms 18371, fol. 82).

31. D. Willemse, op. cit., p. 108 sq.; G. Dulac, « Ribeiro Sanches et le développement de la Russie », art. cit., 1996.

32. Vienne, ms 12714. 
Samoyèdes et les Lapons, et avec le prince Mihail Ščerbatov, historien de la Russie et lui aussi membre de la commission du commerce. Le peu qui subsiste de cette dernière correspondance et quelques échos qu'on en trouve dans les lettres de Sanches à Stählin, montrent qu'il s'agissait d'une relation confiante, en grande partie consacrée à des échanges d'idées et à des nouvelles littéraires, ainsi qu'aux achats de livres dont Sanches se chargeait pour le prince, non sans prendre quelques initiatives en ce domaine : il lui enverra en 1774 La Politique naturelle, ouvrage anonyme du baron d'Holbach, en en commentant les maximes d'une façon, semble-t-il, fort élogieuse que le prince approuvera, non sans soupçonner l'auteur d'être « peu scrupuleux sur la religion ${ }^{33}$.

\section{L’apogée : les années parisiennes (1747-1783)}

À son entrée en France, dans les dernières semaines de 1747, Sanches s'arrêta quelques temps à Strasbourg, une ville qui allait jouer un rôle significatif dans ses relations avec la Russie au cours des années suivantes. Il y rencontra l'historien JeanDaniel Schöpflin ${ }^{34}$, personnalité dominante d'une université luthérienne et bilingue, réputée en Europe à l'égal des meilleures universités allemandes et fréquentée par de nombreux étrangers. Elle devait ce succès à la qualité et à la modernité de ses enseignements, notamment ceux dispensés par la faculté de médecine et celle de droit, qui allait abriter en son sein l' «école diplomatique » constituée par Schöpflin au début des années 1750 pour procurer une formation moderne aux futurs cadres politiques. Au cours de la seconde moitié du siècle, et surtout à partir de 1765, les Russes devaient être de plus en plus nombreux à Strasbourg ${ }^{35}$, les uns étant des étudiants boursiers de l'Académie des sciences de Pétersbourg, et à partir de 1770 d'une fondation Golicyn; les autres des jeunes nobles fortunés fréquentant l'école diplomatique ou certaines institutions périphériques comme l'école d'artillerie. Sanches et Schöpflin, tous deux très hostiles à la tutelle exercée par l'Église catholique sur l'enseignement universitaire ${ }^{36}$, se trouvèrent d'autres idées en commun, notamment sur l'importance de la philosophie, des humanités et particulièrement de l'histoire comme propédeutique à toutes les études spéciali-

33. Lettre du 2/13 mars 1775, Vienne, ms 12714, fol. $34 \mathrm{v}^{\circ}$.

34. Jürgen Voss, Jean-Daniel Schöpflin (1694-1771). Un Alsacien dans 1'Europe des Lumières, Strasbourg, Société savante d'Alsace, 1999.

35. Jürgen Voss, «Les étudiants russes à l'université de Strasbourg au XVIII" siècle », in C. Grau, S. Karp, J. Voss, eds, Deutsch-russische Beziehungen im 18. Jahrhundert. Kultur, Wissenschaft und Diplomatie, Wiesbaden, 1997, p. 351-373.

36. Les papiers de Sanches contiennent plusieurs morceaux inédits sur ce thème. L'un des plus développés est une lettre à Jean-Baptiste Daragon, du collège de Beauvais, qui doit dater de novembre1762 et répond à l'envoi d'une brochure consacrée à l'éducation que Daragon vient de publier: Sanches y fait le procès des universités catholiques, où il lui paraît impossible d'introduire « les connoissances necessaires a la conservation des États », et plaide pour la création d'universités royales débarrassées de toute influence ecclésiastique (Madrid, ms 18373, fol. 33-40). 
sées, y compris médicales, l'histoire moderne devant en outre à leurs yeux tenir une place essentielle dans les sciences politiques et juridiques. Leur correspondance, dont il ne reste que quelques lettres qui s'échelonnent entre 1751 et $1755^{37}$, révèle une véritable amitié et une grande confiance réciproque, bien qu'à l'évidence leurs orientations philosophiques respectives fussent fort différentes : car Schöpflin, disciple de Christian Wolff, en restait aux principes de l'Aufklärung et se montrait par exemple très réservé à l'égard de l'Encyclopédie, alors que Sanches trouvait principalement son inspiration dans les Lumières anglo-saxonnes et françaises. Quoi qu'il en soit, c'est grâce à ces relations que l'université de Strasbourg se trouva au centre de plusieurs projets éducatifs conçus ou soutenus par Sanches qui ne concernèrent pas tous la Russie. Ainsi, par une allusion de Schöpflin qui se disposait à les accueillir, on apprend qu'il projetait en 1752 de faire venir à Strasbourg des Espagnols qui seraient formés dans l'école d'accouchement de Fried, dont la réputation était grande ${ }^{38}$. En 1755, il adressa à Schöpflin un jeune homme de dix-huit ans, Charles de Mertens, pour lequel il avait dressé un plan d'étude comportant un enseignement universitaire préparatoire aux études médicales: docteur en 1758, Mertens devait pratiquer à Vienne, où il était protégé par le prince D. M. Golicyn et peut-être par Van Swieten, avant d'être engagé en 1767 par Ivan Beckoj, sur la recommandation de Sanches, comme médecin de la Maison des enfants trouvés de Moscou $^{39}$. Vers 1756, puis en 1766, il rédigea, comme nous l'avons vu, des plans d'étude conçus pour de jeunes nobles russes, à la demande respectivement du vice-chancelier Mihail Voroncov et du comte Kirill Razumovskij : ce dernier texte ${ }^{40}$, qui est le plus développé, ne traite que de l'aspect proprement russe de la formation des jeunes gens, dont il est précisé que les bases doivent être acquises sous la direction de Schöpflin. Enfin en 1762 et en 1766, Sanches fut invité par le prince Dmitrij Mihajlovič Golicyn, ambassadeur de Russie à Vienne, à donner son avis sur le plan détaillé d'une fondation qu'il voulait établir,

37. Les lettres subsistantes de Schöpflin à Sanches, conservées à Vienne (ms 12713), ont été publiées in Richard Fester, ed., Johann-Daniel Schöpflin, Brieflicher Verkehr, Tübingen, 1906.

38. Ibid., 19 avril 1752, p. 41.

39. Charles de Mertens (1737-1788) réussit à préserver la Maison des enfants trouvés lors de la peste de Moscou (1771) et il décrira l'épidémie dans ses Observationes medicae publiées en 1778 à Vienne, où il s'était installé après avoir quitté la Russie en 1774. Il avait fait la connaissance de Sanches alors qu'il était encore enfant, vers 1749 (Vienne, ms 12713, fol. 627) et il semble avoir entretenu avec lui une correspondance très suivie, mais la partie subsistante ne commence qu'en 1777 (Vienne, ms 12713). C'est par son intermédiaire que Sanches envoyait des livres au prince D. M. Golicyn, qui le protégeait, par exemple le Supplément de 1'Encyclopédie en 1777. Sa mère, son frère et ses sœurs vivaient à Paris.

40. [Pensées sur l'éducation d'un jeune seigneur russe], autographe daté à la fin «Paris 6 novembre 1766 », Bibliothèque publique de Braga, ms 640, fol. 63-90. João Miranda a découvert une copie manuscrite de ce texte en forme de lettre dans les archives des Affaires étrangères, à Lisbonne. L'identification du destinataire que nous proposons (le comte Kirill Razumovskij) nous paraît certaine : elle est notamment fondée sur la phrase indiquant que ses enfants ont été confiés à Schöpflin : voir à ce sujet notre article : «Civiliser la Russie : sept ans de travaux de Ribeiro Sanches », à paraître in G. Dulac et D. Taurisson, eds., La Culture française et les archives russes : une image de l'Europe au XVIII' siècle, Ferney-Voltaire, CIEDS (collection « Archives de l'Est »). 
selon les volontés de sa défunte épouse, pour permettre la formation de jeunes Russes en Occident ${ }^{41}$ : ce projet, qui avait été préparé par le jeune Strasbourgeois Ludwig Henrich Nicolay ${ }^{42}$, que Sanches avait bien connu à Paris en 1760 , et par son disciple Charles de Mertens, reprenait les idées de base du médecin portugais et prévoyait une formation destinée à de futurs médecins ou juristes dans le cadre de l'université de Strasbourg. Sanches entretenait de bonnes relations avec d'autres professeurs de la ville. Ainsi on le consulta en 1752, par l'intermédiaire de Schöpflin, sur un projet du Dr Philippe Henri Böcler concernant l'enseignement de la chirurgie ; et on peut établir indirectement qu'il était l'ami du Dr Jacques Reinbold Spielmann (1722-1783) ${ }^{43}$ : très connu en Europe par ses travaux et les nombreuses correspondances qu'il entretenait dans le monde scientifique, il est mentionné dans les procès-verbaux de l'Académie de Pétersbourg pour l'assistance qu'il apportait aux étudiants russes ${ }^{44}$.

Dès son arrivée à Paris en décembre 1747, Sanches agit comme correspondant de l'Académie des sciences de Pétersbourg, jusqu'à ce qu'il apprenne au début de 1749 qu'il en a été exclu sur l'ordre de l'impératrice. Razumovskij l'informera plus tard du motif de cette décision, qui soulèvera des protestations, notamment celle de L. Euler : Élisabeth avait été sensible aux accusations des ennemis de Sanches qui prétendaient qu'il « judaïsait », tout en se prétendant catholique. Pendant ces quelques mois, il établit rapidement des relations avec les plus grands savants de l'époque et avec des personnalités parmi les plus connues ${ }^{45}$ : Delisle, bien sûr, qu'il a connu en Russie, mais aussi Buffon et Camille Falconet, qu'il présentera tous deux comme des amis, Dortous de Mairan, l'abbé Nollet, Jussieu, Réaumur, d'Alembert, d'Anville... Il sert d'intermédiaire entre les deux académies des sciences, transmettant des propositions et des questions, des nouvelles littéraires et

41. Je remercie vivement Wladimir Berelowitch de m'avoir communiqué les pièces essentielles du dossier de cette fondation, conservé aux Archives d'actes anciens à Moscou (RGADA, f. 1263, op. $1, n^{\circ}$ 6882). Établie à titre privé grâce à un legs de la princesse E. D. Golicyna, mais approuvée officiellement par un oukaze de l'impératrice, elle devait être placée sous le contrôle de la direction de la Maison des enfants trouvés, donc de Beckoj, qui eut à donner son avis. Sanches avait recommandé que les candidats retenus pour mener des études médicales avec son soutien soient issus de le petite noblesse et qu'ils arrivent très jeunes à Strasbourg pour y passer neuf ou dix ans. Il ne sera que partiellement suivi.

42. L. H. Nicolay (1737-1820) sera engagé en 1766 par le comte K. Razumovskij comme tuteur et compagnon de voyage de son fils aîné Aleksej; en 1769, il sera appelé à Pétersbourg pour être un des tuteurs du grand-duc Paul, dont il deviendra en 1773 le secrétaire privé ; à cette époque il correspondait, semble-t-il, assez régulièrement avec Sanches (d'après les lettres de celuici à Stählin); en 1798 enfin, il devait être nommé président de l'Académie impériale des sciences. Voir Edmund Heier, L. H. Nicolay and his contempories, La Haye, Nijhoff, 1965.

43. Dans une lettre à Spielmann, datée de Londres, 21 octobre 1783 et arrivée à Strasbourg après la mort du destinataire, J. H. Magellan écrit : « Notre ancien respectable ami le Dr Sanchez vient de mourir à Paris le 14 du courant [...]. » (Théodore Vetter, « Les relations scientifiques entre la Grande-Bretagne et l'Alsace dans la seconde moitié du XVIII ${ }^{\mathrm{e}}$ siècle. À propos d'une lettre inédite de Jean-Hyacinthe de Magellan, F.R.S., au professeur strasbourgeois Jacques Reinbold Spielmann », Revue d'Alsace, 115, 1989, p. 37-67.

44. Procès-verbaux, t. 2, p. 497 (avril 1763).

45. Ces lettres sont principalement conservées parmi la correspondance scientifique, aux Archives de l'Académie des sciences de Pétersbourg (f. 1, op. 3). 
des livres, ainsi que des comptes rendus de séances de l'Académie royale. C'est lui par exemple qui propose le géographe d'Anville comme membre étranger de l'Académie de Pétersbourg et négocie avec lui l'acquisition de cartes. Il transmet à plusieurs reprises des questions de Buffon, et fait notamment parvenir au gouverneur d'Astrakhan celles qui concernent la faune de la mer Caspienne, ou sert d'intermédiaire entre l'auteur de l'Histoire naturelle et Klingstedt, à propos des Samoyèdes. Dans cette activité, il est surtout en correspondance avec les responsables de la chancellerie et de la bibliothèque de l'Académie impériale, Johann Daniel Schumacher, Johann Caspar Taubert et Gregorij Teplov, alors assesseur, dont il écrira des années plus tard qu'il était son « ancien ami » : il semble en effet qu'il ait gardé avec lui des relations, alors qu'il était devenu secrétaire de Catherine II. À partir de 1763, après sa réintégration à l'Académie, Sanches reprend son activité de correspondant avec beaucoup de zèle, et comme en témoigne l'agenda qu'il commence à ce moment-là pour en tenir registre, elle reste très soutenue jusqu'en 1770 , et se poursuit jusqu'à la fin de sa vie. À Petersbourg, ses partenaires seront, pendant une vingtaine d'années, outre les fonctionnaires de la chancellerie, les secrétaires successifs, Gerhard Friedrich Müller, Jacob von Stählin, et enfin Johann Albrecht Euler, à partir de 1769. Mais il entretient parallèlement une correspondance personnelle avec plusieurs des académiciens qu'il avait connus à Pétersbourg : il ne nous reste de vestiges importants que de celle qu'il échange avec Stählin jusqu'à la fin de sa vie ${ }^{46}$.

La seconde sphère de relations que Sanches développe à Paris est constituée de personnages de la cour de Pétersbourg qu'il a connus pour la plupart avant son installation en France : comme nous l'avons signalé, il entretient une correspondance avec un petit nombre d'entre eux, et surtout il rencontre la plupart de ceux qui séjournent à Paris. Ainsi le chargé d'affaires russe à Paris, le conseiller Fedor Dmitrievič Behteev, qui est en relations amicales avec lui, mentionne ses visites et transmet de ses nouvelles dans ses dépêches de 1756-1757 au vice-chancelier Mihail Voroncov ${ }^{47}$, qui de son côté conseillera à Ivan Šuvalov de s'en tenir aux avis de l'ancien médecin de la cour ${ }^{48}$. Les cahiers de notes de Sanches prouvent qu'à cette époque il s'intéresse de très près aux événements qui marquent l'évolution du pays : la création de l'université de Moscou, en 1755, lui inspire par exemple des réflexions critiques qui datent, semble-t-il, de l'année suivante et ont peut-être été adressées au vice-chancelier. Mais bientôt certaines de ces relations vont prendre une consistance nouvelle, notamment sous l'effet de la fièvre de projets qui caractérise les premières années du règne de Catherine II. À la fin de 1756 arrivent à Paris trois personnes que nous avons déjà eu l'occasion de mentionner et qui vont avoir à cet égard une importance durable dans

46. Vienne, ms 12713 pour les lettres reçues de Stählin (1773-1779) ; Pétersbourg, Bibliothèque nationale de Russie, fonds 871, $\mathrm{n}^{\circ} 244$, minutes des lettres de Stählin (1769-1780); $\mathrm{n}^{\circ} 664$, lettres de Sanches (1770-1777) ; Pétersbourg, Archives de l'Académie des sciences, f. 1, op. 3 (Correspondance scientifique), $\mathrm{n}^{\circ} 49,50$, etc.

47. Arhiv Knjazja Voroncova/Archives du prince Vorontsov, Moscou, Ed. A. I. Mamontov, 1870-1895, t. 3, p. 199, 228, 236, 237, 258, etc.

48. Ibid., t. 32, p. 65 (février 1766). 
l'activité de Sanches et ses relations avec la Russie : ce sont le prince Dmitrij Mihajlovič Golicyn (1721-1793), qui prendra provisoirement la tête de la mission russe à Paris en 1760-1761 avant d'être nommé à Vienne ; sa femme, Ekaterina Dmitrievna, née Kantemir ${ }^{49}$, qui mourra à Paris en 1761 ; et l'oncle de la princesse, Ivan Ivanovič Beckoj, qui fait plusieurs séjours prolongés à Paris avant d'être rappelé en 1761 à Pétersbourg ${ }^{50}$, où il deviendra quelques années plus tard le proche collaborateur de Catherine II pour les établissements d'éducation. Sanches est le médecin et l'ami de ce petit groupe, auquel se joint le jeune prince Dmitrij Alekseevič Golicyn (17341803), volontaire dans l'armée française pendant la guerre de Sept Ans, puis attaché à l'ambassade russe en 1760 , au seuil d'une carrière diplomatique qu'il va poursuivre à Paris jusqu'à la fin de 1767, puis à La Haye à partir de 1770. Dans la série de dépêches où il argumentera, à partir d'octobre 1765, en faveur de la libération des paysans russes, il se présentera comme un disciple de Sanches, qui de son côté, dans les mémoires qu'il rédigera à la demande de Beckoj à partir de 1764, mentionnera le prince D. M. Golicyn et la façon dont il avait aménagé la condition de ses paysans. Comme nous l'avons vu, l'affaire de la fondation, qui devait traîner de 1762 à 1770, réunit en partie les mêmes acteurs.

Les relations portugaises de Sanches se développent dans les années 1750, et surtout après l'arrivée au pouvoir de Pombal au milieu de la décennie, pour des raisons qui ne sont pas sans analogies avec ce que nous venons d'évoquer pour le côté russe de son activité : d'une part le fait que d'assez nombreux Portugais, médecins, savants et diplomates, qui séjournent à Paris voient en notre médecin une personnalité particulièrement intéressante ; de l'autre, son association aux réformes qui se préparent au Portugal dans tous les domaines de la vie culturelle, sociale et économique. Cette symétrie est cependant trompeuse car, contrairement à ce qui se produira pour les écrits destinés à Beckoj, et en fin de compte à Catherine II, la consultation de Sanches sur les affaires portugaises prend un tour officiel et quasi public, puisque plusieurs des travaux qui lui sont commandés sont imprimés à Paris aux frais du gouvernement de Lisbonne. Aussi a-t-il à cette époque de fréquents contacts avec l'ambassadeur du Portugal à Paris, Pedro da Costa de Almeida Salema, ainsi qu'avec son ami et protecteur Luis da Cunha, neveu et homonyme du diplomate déjà mentionné. Mais, laissant de côté ces relations proprement politiques, nous dirons quelques mots de deux hommes qui, liés à Sanches par une amitié durable, auront dans son activité intellectuelle une place qui illustre assez bien la nature et le fonctionnement des réseaux dont il est alors entouré. José Joaquim Soares de Barros (17211793), après avoir étudié les mathématiques en Angleterre et en Hollande, s'installe en 1750 à Paris, avec le soutien financier du gouvernement portugais ${ }^{51}$. Élève de Clairaut et de Delisle, il publie en français des travaux qui assurent sa réputation scienti-

49. Elle était la fille d'A. I. Troubeckaja, demi-sœur de Beckoj, et du prince Dmitrij Kantemir.

50. Petr Mihajlovič Majkov, Ivan Ivanovič Beckoj. Opyt ego biografii, Saint-Pétersbourg, Utilité publique, 1904, p. 31 sq.

51. Maximiano Lemos, « Amigos de Ribeiro Sanches », Archivo historico portuguez, 8, 1911, p. 281-295 et 447-469, ici p. 450-469 ; Antonio Ferrão, Ribeiro Sanches e Soares de Barros : novos elementos para as biografias desses académicos, Lisbonne, Ottosgrafica, 1936. 
fique, et il est associé aux académies de Paris et de Berlin. Sanches lui rend un hommage appuyé dans son traité d'hygiène public, imprimé à Paris en $1756^{52}$ : c'est que Soares de Barros s'intéresse comme lui à l'application des mathématiques aux sciences sociales. Rentré à Lisbonne en 1760, il revient presque aussitôt à Paris, chargé d'une mission diplomatique qui tourne mal : disgracié, il quitte définitivement Paris en juillet 1761 et se retire au Portugal. Il y reçoit le soutien de Sanches et se consacre désormais à des recherches tournées vers l'économie, la démographie et les statistiques appliquées à la vie humaine, disciplines auxquelles son ami, au même moment, emprunte plus d'un argument dans ses mémoires sur le développement de la Russie. Un épisode de leurs relations qui se situe entre 1763 et 1769 montre comment peuvent converger les relations de types très différents qu'entretient Sanches : quand Soares de Barros lui fait parvenir une petite quantité d'une terre trouvée à Maffra qui semble avoir des propriétés anticancéreuses, celui-ci procède à des essais et y associe Gaubius, à Leyde ; il informe d'autre part son ami le prince D. A. Golicyn, qui sollicite par l'intermédiaire du consul de Russie à Lisbonne l'autorisation d'exporter du Portugal de la terre de Maffra, mais il se heurte à un refus, que Gaubius déplore en 1768 ; l'année suivante, Catherine II, au cours d'une audience qu'elle accorde à Golicyn, lui exprime son vif désir de voir les recherches se poursuivre et son espoir que le marquis de Pombal les favorisera ; il s'ensuit un nouvel échange de lettres avec Sanches ${ }^{53}$, mais le refus des autorités de Lisbonne sera confirmé, et les essais ne donneront que des résultats douteux ${ }^{54}$. C'est également, semble-t-il, au début des années 1750 que Sanches se lie avec Gonsalo Xavier d'Alcaçova ${ }^{55}$, avec qui il entretiendra jusqu'à la fin de sa vie une correspondance régulière et riche en informations, autant qu'on en puisse juger par les lettres subsistantes, qui vers 1764 se succèdent généralement au rythme de deux par mois. D'Alcaçova, littérateur dilettante et homme éclairé proche de l'entourage de Pombal, était alors le secrétaire de l'Académie royale d'histoire. Il évoque par exemple dans ses lettres de 1765 l'ouverture du Collège des nobles, dont Sanches avait contribué à préparer l'organisation dans ses Cartas sobre a educação de mocidade, publiées en 1760, alors que l'expulsion des jésuites venait d'ouvrir la voie à l'instauration d'un système éducatif dépen-

52. «Tratado da conservaçao da saude dos povos, em Paris, e se vende em Lisboa, em casa de Bonardel e Du Beux, 1756 », in Ribeiro Sanches, Obras, op.cit., t. 2, p. 156. Sanches souligne également ce qu'il doit à Soares de Barros dans un paragraphe inséré avant ses «Consideraçoins sobre os terramotos » placées à la fin de l'ouvrage (op. cit., p. 363). Nous ne nous étendrons pas sur les relations que Sanches entretient au Portugal à cette époque de sa vie : il faudrait y faire figurer par exemple le Dr João Mendes Sachetti Barbosa (1714-avant 1780), médecin à l'hôpital d'Elvas, en relation avec Castro Sarmento à Londres et associé à la Royal Society en 1755 : il correspond longuement avec Sanches à partir des années 1750.

53. Moscou, Archives d'actes anciens (RGADA), f. 1263, op. 1, n 1117, fol. 53, 55-56, etc. ; également Braga, ms cité, fol. 133 (copie partielle de la main de Sanches d'une lettre de D. A. Golicyn datée d'août 1769 et écrite à Pétersbourg après une entrevue avec l'impératrice).

54. Sur cette affaire, voir notamment, João Miranda, « A. N. Ribeiro Sanšes i russko-Portugal'skie svjazi... », art. cit., p. 48.

55. [Anonyme], « Gonsalo Xavier d'Alcaçova Carneiro e cartas da sua correspondencia particular com Antonio Nunes Ribeiro Sanches », Revista Michaelense, Anno 2, 1919, p. 382-410, 501-529. 
dant uniquement du pouvoir civil ${ }^{56}$. Un des aspects les plus intéressants de cette correspondance réside par ailleurs dans les commentaires suscités par les nouvelles littéraires ainsi que les livres et périodiques que d'Alcaçova reçoit en grand nombre de Sanches. Il est ainsi beaucoup question de la sortie des derniers volumes de l'Encyclopédie et de celle du Supplément. Notons seulement à ce propos un détail très caractéristique de la position de l'informateur : le 12 mai 1767, d'Alcaçova fait écho à des confidences de Diderot, transmises par Sanches, sur les retranchements opérés à son insu par le libraire Le Breton dans les articles philosophiques des derniers volumes du Dictionnaire raisonné ${ }^{57}$ : l'affaire était encore tenue secrète, et Grimm ne la révèlera dans sa Correspondance littéraire qu'en janvier $1771^{58}$.

Ce petit épisode nous amène à considérer une dernière sphère des relations de Sanches à Paris : elle concerne le milieu philosophique, et tout particulièrement Diderot et certains de ses amis. Malgré son importance, elle est restée jusqu'à présent à peu près ignorée, probablement parce que ni les correspondances, ni les mémoires qui attestent de la vie des salons, où Sanches ne paraissait guère, ne peuvent nous renseigner à son sujet. Par rapport à ce milieu, notre médecin a probablement occupé une place un peu marginale, ce qui ne l'a pas empêché d'entretenir des relations significatives et, semble-t-il, assez régulières avec le directeur de l'Encyclopédie, au moins à partir de 1760. C'est le 28 octobre de cette année-là, depuis le Grandval, résidence de campagne du baron d'Holbach, que Diderot présente à Sophie Volland « le docteur Sanchez, cy devant premier médecin de la czarine, juif de religion et portugais d'origine », en précisant qu'il s'agit d' «un homme bien précieux $»^{59}$. Il ne le mentionnera de nouveau qu'en 1767 , en faisant à Etienne Falconet l'apologie de L'Ordre naturel et essentiel des sociétés politiques, de Le Mercier de la Rivière, ouvrage, écrit-il, qui a fait forte impression sur le «docteur Sanchez, qui n'est certainement ni un ignorant ni une petite tête " ${ }^{60}$. À ces deux mentions élogieuses, mais en elles-mêmes sans grande portée, les archives personnelles de Sanches apportent d'utiles compléments. On trouve tout d'abord dans un volume conservé à Madrid la copie, de la main de Sanches, d'un texte dont il précise que c'est Diderot qui le lui a communiqué, au début de 1761 : il s'agit

56. Dans une lettre à Sanches du 25 mars 1765, Alcaçova note avec satisfaction que parmi les maîtres du collège, qui sont pour la plupart italiens, les ecclésiastiques sont peu nombreux (« Gonçalo Xavier d'Alcaçova Carneiro », ibid., p. 515). À propos de ces lettres sur l'éducation de la jeunesse, voir Maximiano Lemos, Ribeiro Sanches..., op. cit., p. 260 sq. Imprimé à Paris à une cinquantaine d'exemplaires, l'ouvrage porte une dédicace dont le destinataire n'est pas nommé : il s'agit vraisemblablement de Salema (Sanches, Obras, op. cit., t. 1, p. 368-369).

57. «O que V. Mce me diz a respeito da Enciclopedia communicado por Diderot, me desgosta bastante ; e bem percebia eu em muitos artigos que tenho folheado, quanto estão diminutos [...] » écrit assez naïvement d'Alcaçova, qui espère que ces lacunes seront comblées par un supplément (art. cit., p. 518).

58. F. M. Grimm, Maurice Tourneux, ed., Correspondance littéraire, Paris, Garnier, 18771882, t. 9, p. 203-215. Sur cet épisode, voir Jacques Proust, Diderot et l'Encyclopédie, Paris, Colin, 1982,p. 70 .

59. Diderot, G. Roth et J. Varloot, eds, Correspondance, Paris, Minuit, 1955-1970, t. 3, p. 202.

60. Diderot, Correspondance, ibid., t. 7, p. 79. 
d'une longue lettre du philosophe à son frère dont le texte sera repris dans l'article INTOLÉRANCE de l'Encyclopédie (à moins que la relation ne soit inverse) ${ }^{61}$. Vient à la suite, datée du 26 mars 1761, la minute de documents destinés à Diderot qui prouvent que c'est ce dernier, et non d'Alembert, comme on l'a cru, qui a engagé Sanches à écrire le seul article qui porte son nom dans l'Encyclopédie. Une note insérée dans le même volume pourrait même indiquer qu'un autre article avait été demandé au médecin portugais ${ }^{62}$. En poursuivant cette chronique, on rappellera les confidences de Diderot dont Sanches fait état en 1767 dans une lettre à d'Alcaçova. C'est à partir de 1768, semble-t-il, qu'on rencontre fréquemment dans les cahiers de Sanches des notes prises sur les ouvrages antichrétiens du baron d'Holbach, tout d'abord Le Christianisme dévoilé et La Contagion sacrée, ou Histoire naturelle de la superstition ${ }^{63}$, puis en 1771 l'Essai sur les préjugés ${ }^{64}$, qui s'en prend aux «despotes » et irritera fortement Frédéric II. Il est évident que Sanches est en mesure de suivre de très près la production du baron, et l'on comprend aisément pourquoi quand on découvre, parmi ses notes, le résumé d'un billet destiné à Diderot et daté du 29 août 1772 : il y annonce au philosophe qu'il vient de recevoir du prince Golicyn (certainement celui de La Haye) Le Bon sens (de d'Holbach), et qu'il va le lui apporter. Deux ans plus tard, comme on l'a vu, il enverra à Pétersbourg La Politique naturelle, toujours du même auteur, et en copiera des extraits dans ses cahiers ${ }^{65}$. On peut en conclure que Sanches, non content d'être un grand lecteur de ces ouvrages prohibés, a probablement constitué avec le prince D. A. Golicyn une filière qui leur permettait de les faire pénétrer aisément en France, peut-être par l'intermédiaire de la mission russe à Paris ${ }^{66}$. Bien d'autres indices de ses relations avec Diderot et son entourage existent dans ses archives, par exemple telle anecdote qu'il tient du philosophe, ou la manière

61. Lettre à l'abbé Diderot du 29 décembre 1760 : Madrid, ms 18372, fol. 316-319; Diderot, Correspondance, op. cit., t. 3, p. 283-288 ; Diderot, John Lough et Jacques Proust, eds, Euvres complètes, Paris, Hermann, 1975, t. 7, « Encyclopédie », p. 541-546.

62. Madrid, ms 18372, fol. 319-323 vº il s'agit d'une minute autographe qui comporte le début d'une lettre de Sanches à Diderot, suivi de la majeure partie du texte qui sera publié dans le dernier volume de l'Encyclopédie sous l'entrée VÉROLE, grosse : « À Monsieur Diderot Autor de l'Encyclopedie Mars 26/ 1761 Vous voules avoir mes pensees sur la Maladie Venerienne chronique, peut etre, incité par la nouveaute que je lui donne, plutot que par l'utilite qui en pourra retirer l'humanité. \& ». Dans ce même volume 18372, fol. 131, se trouve une note de Sanches intitulée « Melancholia / Pour ecrire l'article dans l'Encyclopedie », qui semble dater de 1754 : il a donc peut-être été invité à apporter cette contribution au Dictionnaire, mais l'article correspondant ne porte pas sa signature, contrairement à l'article VÉROLE.

63. Paris, ms 2017, fol. 253.

64. Paris, ms 2015, fol. 68-69.

65. Ibid., fol. $181 \mathrm{v}^{\circ}$.

66. Rappelons à ce propos que lorsque le prince donnera une réédition du Nakaz de Catherine II, qui était interdit en France, il se vantera de pouvoir en introduire en France autant d'exemplaires qu'il le désirait (Moscou, RGADA, f. 1263, op. 1, n 1119, fol. 32, 15 février 1771 ; voir G. Dulac et Ludmilla Evdokimova, art. cit., notamment p. 384-385). 
chaleureuse dont il le recommande à Stählin en 1773, avant son arrivée à Pétersbourg67 ; ou encore les services qu'il rend à Raynal, dont il transmet à Pétersbourg les questions sur l'armée russe, et à qui il procure un mémoire sur le commerce du Portugal, pour son Histoire des deux Indes. Nous nous en tiendrons, pour terminer sur ce point, à un fait plus important : la filiation à peu près certaine qui relie certaines des vues exprimées par Diderot dans les années 1770 sur le processus de «civilisation » de la Russie, aux mémoires écrits par Sanches quelques années auparavant. Car au delà des principes fondamentaux qui leur sont communs, on reconnaît dans certaines pages du philosophe des arguments inspirés à l'ancien médecin de la tsarine par une connaissance précise de la société russe qu'il était bien seul à posséder parmi les encyclopédistes ${ }^{68}$.

\section{Du bon usage des réseaux}

Les relations, et particulièrement les correspondances, entretenues et développées par Sanches ont eu quelques fonctions évidentes et banales, qui méritent cependant attention, à cause du caractère systématique et de l'efficacité qu'il a su leur donner. Leur fonction de base est de faciliter l'acquisition du savoir : ainsi il est très significatif que, dans la lettre à Joachim Pedro de Abreu déjà citée ${ }^{69}$, il mette sur le même plan, pour prouver sa compétence en matière d'enseignement médical, la fréquentation de plusieurs universités européennes, sa connaissance des hôpitaux de Londres et de Russie, et enfin une demi-douzaine de correspondances de longue durée avec d'éminents médecins et naturalistes. Cette fonction traditionnelle des correspondances savantes, qui permettent de collecter des faits, des observations et des informations bibliographiques, voire des livres et d'autres objets, tout en échangeant des idées, a été étendue par Sanches aux différents domaines auxquels il portait intérêt. C'est ainsi qu'il a associé plusieurs de ses correspondants, médecins, mais aussi historiens et érudits, à la collecte d'informations sur les origines historiques de la maladie vénérienne, et que, sur d'autres plans, il a réuni des données sur le commerce portugais ou demandé à Beckoj de lui procurer les statuts des grands établissements d'enseignement créés en Russie. Ce mode de travail suppose des échanges, et on voit en effet Sanches les multiplier, en s'efforçant à son tour de procurer à ses amis informations et publications : ajoutons aux exemples donnés plus haut, celui des services qu'il rendait aux bénédictins de la congrégation de Saint-Maur, ses voisins de la rue des Blancs-Manteaux, dont il fréquentait la biblio-

67. G. Dulac, « Diderot et deux académiciens de Pétersbourg », Europe, 661, 1984, p. 84-93. En fait Stählin prendra une part active aux menées dirigées contre Diderot à partir de l'Académie : voir G. Dulac, « Un nouveau La Mettrie à Pétersbourg : Diderot vu de l'Académie impériale des sciences », Recherches sur Diderot et sur l'Encyclopédie, 16, 1994, p. 19-43.

68. Voir à ce sujet notre article, « Diderot et le "mirage russe” : quelques préliminaires à l'étude de son travail politique de Pétersbourg », Sergueï Karp et Larry Wolff, eds, Le Mirage russe au XVIII ${ }^{\mathrm{e}}$ siècle, Ferney-Voltaire, CIEDS, 2001, p. 149-192.

69. Paris, ms 2019, fol. $168 \mathrm{v}^{\circ}$. 
thèque, et pour qui il lui arrivait de faire venir des ouvrages de Pétersbourg, ou de traduire des documents de l'espagnol ${ }^{70}$. Nous ne connaissons qu'en partie l'étendue qu'il a donnée à ce rôle d'intermédiaire, mais il a été en tout cas important auprès de plusieurs académies, et surtout de celle de Pétersbourg. Pour s'en tenir aux relations privées de Sanches, il faut souligner quelques faits qui leur donnent une portée particulière. Tout d'abord il a, pendant une grande partie de sa vie, été proche d'hommes qui, dans des domaines différents, étaient eux-mêmes à la tête de réseaux étendus, comme Magellan, Schöpflin, Van Swieten ou le prince D. A. Golicyn, ce qui a considérablement accru les dimensions de son propre univers. Lui-même, d'autre part, semble avoir fréquemment favorisé l'extension, au profit de ses disciples et amis, des réseaux sur lesquels il appuyait son activité : c'est ainsi que D. A. Golicyn, Ch. de Mertens et sans doute J. G. Günz ont pu, grâce à lui, entrer directement en contact avec Magellan pour l'acquisition de publications anglaises. Vers 1775-1777, par exemple, il se concerte avec son ami, le médecin et économiste Lebègue de Presle, à propos des livres qu'ils se font envoyer chez Lavoisier ${ }^{71}$ : parmi eux, à côté d'ouvrages de médecine, The Common sense de Thomas Paine, The Wealth of nations, d'Adam Smith, et On Civil liberty, de Richard Price. Enfin, précisément en ce qui concerne l'importation par les soins de Sanches de livres publiés en Grand-Bretagne, on ne peut qu'être frappé par son caractère massif, dès le début des années 1750 et pendant les décennies suivantes, dans ses deux domaines de prédilection, la médecine, et surtout la philosophie politique, celle des Écossais notamment ${ }^{72}$ : aussi peut-on soupçonner que cette activité, prolongée par un travail intellectuel souvent novateur, a pu lui conférer, auprès de certains secteurs de la république des lettres, un rôle qui allait au delà de celui d'un simple intermédiaire.

Au milieu des réseaux que nous avons évoqués, Sanches occupe une position qu'on est tenté de juger exceptionnelle, parce qu'il y entretient des liaisons très actives et confiantes avec des milieux intellectuels très différents, voire opposés : à cet égard, l'attitude qu'il a adoptée paraît bien éloignée de celle d'un Formey, par exemple, dont l'énorme correspondance ${ }^{73}$, adressée à des centaines d'interlocuteurs, donne l'image d'un univers intellectuel très homogène, et même relativement limité. Au contraire, entre certains des correspondants les plus amicaux de Sanches, les oppositions sont telles qu'on pourrait le soupçonner d'une sorte de duplicité. Il maintient ainsi pendant des années des échanges, qui ne se limitent pas aux ques-

70. Paris, Bibliothèque nationale de France, $\mathrm{ms}$ fr 9356 , fol. $198 \mathrm{r}^{\circ}-\mathrm{v}^{\circ}, 200,201,203$ : dans ces documents sans date (mais certains ne sont pas antérieurs à 1777) sont nommés dom Brial, dom Clément, dom Vidal.

71. Vienne, ms 12713, fol. 594, etc. Les lettres de Lebègue de Presle ne sont pas toujours datées.

72. Au début des années 1750 , ce sont les œuvres philosophiques et historiques de Hume qui figurent en grand nombre dans ses journaux et recueils de notes; plus tard, on y rencontre des ouvrages de James Steuart, Adam Fergusson, William Robertson, John Millar, Adam Smith, etc.

73. André Bandelier, «Sur la correspondance de Formey », in Benoît Melançon, dir., Penser par lettres, Québec, Éditions Fides, 1998, p. 205-217. 
tions scientifiques, avec des hommes comme les Euler, père et fils, Stählin, ou Schöpflin, hostiles au mouvement philosophique français de la seconde moitié du siècle : il accueille par exemple sans s'émouvoir l'indignation de Schöpflin contre « les philosophes licencieux de Berlin », cette «secte» française dont Christian Wolff s'est plaint auprès de l'historien strasbourgeois qu'elle lui a fait perdre l'estime du roi de Prusse. Cependant il noue bientôt d'excellentes relations avec les encyclopédistes les plus radicaux, et se trouve visiblement en accord avec les arguments antichrétiens du baron d'Holbach ou de Diderot. Il semble en fait qu'à un intérêt pragmatique pour ce que des correspondants très divers peuvent lui apporter, s'ajoute chez lui un esprit d'ouverture et de tolérance qui a pu contribuer à lui donner une vision très large de la vie intellectuelle européenne.

Si l'on considère d'autre part le rôle de consultant que Sanches a plusieurs fois été amené à jouer, il est manifeste que sa curiosité toujours en éveil, éclairée par une grande culture philosophique et politique, lui a procuré une bonne connaissance de plusieurs sociétés très différentes et de leurs institutions. Aussi a-t-il souvent adopté des perspectives élargies aux dimensions du continent : il est souvent question de l'Angleterre et de la France dans ses écrits sur la Russie, et il emprunte des exemples à la Russie et à l'Italie dans ses ouvrages destinés au Portugal. Ses divers réseaux ont été utilisés pour tenir à jour ces connaissances, notamment en ce qui concerne la Russie, le Portugal, et sans doute aussi l'Italie (il s'est particulièrement intéressé aux réformes engagées dans le grand-duché de Toscane et dans le royaume de Sardaigne), lui permettant de mettre en œuvre, à des fins pratiques, cette méthode comparative qu'illustrent les travaux de l'école historique écossaise. Si l'existence de réseaux de relations en amont de ses travaux est importante, elle ne l'est pas moins en aval, car elle a permis, concernant par exemple la Russie, une certaine diffusion, indirecte ou différée, du résultat de ses recherches. Les mémoires pour le développement et la « civilisation » de la Russie qu'il a adressés à Beckoj n'ont certes guère produit de résultats, sinon mineurs. Mais les idées qu'il a lancées n'en sont pas pour autant restées totalement confinées: elles ont été reprises par le prince D. A. Golicyn, et aussi par Diderot qui a exploité certaines d'entre elles, aussi bien dans ses notes destinées à Catherine II que dans les pages écrites pour l'Histoire des deux Indes. Peut-être même faut-il prendre en considération le fait que les minutes de ses mémoires les plus radicaux sur la Russie aient été recueillies après sa mort par les Voroncov, probablement par Semen Romanovič, le plus jeune des neveux du chancelier M. I. Voroncov avec qui Sanches avait été particulièrement lié : c'était vers la fin du siècle, et le souvenir de ses travaux n'était pas totalement effacé, pas plus que n'étaient véritablement périmés les diagnostics qu'il avait portés sur l'état du pays. Enfin la présence dans les archives portugaises de plusieurs écrits importants de Sanches consacrés à la Russie suggère que, de ce côté-là également, les liaisons qu'il avait eues leur ont assuré une certaine audience : ils traitaient de l'éducation des nobles, de la manière de s'attacher les pays conquis (ou les colonies), du rôle des couches populaires dans le développement d'un pays, et les principes qu'ils illustraient n'étaient pas indifférents aux Portugais éclairés. 
Pour conclure, je formulerai rapidement trois constatations qui me semblent présenter un certain intérêt méthodologique, parce que le cas de Sanches est particulièrement significatif à cet égard. Tout d'abord, il n'est peut-être pas inutile de souligner, à partir de cet exemple, à quel point l'étude des correspondances subsistantes peut, à elle seule, se révéler insuffisante, voire trompeuse, pour l'étude des relations intellectuelles : car on sera souvent tenté de tirer des conclusions des lacunes qu'elles comportent, bien qu'elles ne soient qu'accidentelles. Mais il est vrai qu'on ne dispose que rarement, pour corriger une telle erreur de perspective, d'archives de travail aussi importantes que dans le cas de notre médecin. En second lieu, il me semble que l'imbrication des différentes relations internationales que Sanches a entretenues toute sa vie est suffisament marquée et significative pour que l'on évite d'en isoler certaines de l'ensemble : c'est pourtant ce qui s'est souvent produit dans les études qui lui ont été consacrées, le côté russe et le côté portugais de ses activités étant considérés séparément, tandis que d'autres, français notamment, étaient à peu près négligés. Enfin on peut faire une remarque analogue à propos des deux versants, scientifique et politique, de ses travaux et de ses échanges intellectuels, en rappelant que lui-même a toujours établi de multiples rapports entre eux, même s'ils correspondaient le plus souvent, autour de lui, à des réseaux de relations distincts : car, et ce ne sont là que deux raisons parmi d'autres, la médecine dont il traite le plus volontiers est ce qu'il appelle la « médecine politique » (c'est-à-dire l'hygiène publique, ou la définition de formations médicales adaptées au cadre national); et d'autre part les conditions du développement des sciences dans un pays constituent précisément un des problèmes dont il part pour aborder tous les autres, sociaux, économiques, voire institutionnels, qui se posent à un État, qu'il soit «gothique » comme le Portugal, ou «militaire » comme l'empire russe (pour reprendre ses qualificatifs).

Centre d'étude du XVIII siècle

Université Paul-Valéry, route de Mende, 34199 Montpellier CEDEX 5 georges.dulac@univ-montp3.fr 